\title{
Full 3D Rigid Body Automatic Motion Correction of MRI Images
}

\author{
Yi Su, Armando Manduca, Clifford R. Jack, E. Brian Welch, and \\ Richard L. Ehman \\ Mayo Clinic, Rochester, MN 55905 USA
}

\begin{abstract}
We demonstrate the first successful automatic motion correction of 3D MRI data with rigid body motion in all six degrees of freedom. An existing $2 \mathrm{D}$ retrospective technique is extended to $3 \mathrm{D}$ with a shear-based factorization of 3D rotations and simultaneous optimization of motion corrections in all six degrees of freedom. Tests on motion corrupted 3D brain images from elderly research subjects show dramatic improvements in image quality.
\end{abstract}

\section{Introduction}

In several ongoing research studies here, high resolution 3D MRI brain scans are required of elderly, often cognitively impaired individuals. This population is predisposed to motion and many subjects are simply unable to hold sufficiently still for the length of the exam. Some form of motion correction is thus imperative. Recently, automatic methods based on iterative optimization of an image quality cost function have been proven effective for 2D rigid body motions [1,2]. However, such schemes require inverse FFTs of the entire data set at each iteration, as well as regridding in $\mathrm{k}$-space for each trial rotation, making them computationally intensive. They are also susceptible to local minima, being essentially optimization techniques in a very high dimensional space. Extrapolation of these techniques to 3D data sets and motions has not been attempted until now due to the computational requirements and the increased dimensionality of the search space.

\section{Methods}

The data obtained in our studies are 3D SPGR acquisitions with TR/TE $=23 / 9$ msec. The k-space dimensions are $256 \times 144 \times 124(22 \times 16.5 \times 19.8 \mathrm{~cm}$ FOV), acquired in xzy order. We assume no motion during the acquisition of each $\mathrm{xz}$ slab $(2.85 \mathrm{sec})$, reducing the dimensionality of the correction process to six degrees of freedom that specify the subject's position at each of 144 points in time. The xz slabs are considered in blocks, with the block size initially 72 slabs and progressively halved until slabs are considered individually. At a given block size, blocks are optimized individually, working outward from DC. These six-dimensional optimizations are performed with a simplex algorithm; at each iteration with a trial set of motion 
parameters the corresponding corrections are applied to the block under consideration, the entire data set is reconstructed, and the image quality is evaluated. Blocks not currently under consideration have their parameters fixed at the last values found. The cost function is the entropy of the image gradient [2], and 3D rotations are efficiently performed by a factorization into 4 shear operations [3].

\section{Results}

It is clear that the automatic motion correction dramatically improves the quality of the images (Fig. 1). While further improvement may be possible, the data is now usable for research purposes and would even be suitable for diagnosis. This represents the first successful automatic motion correction of 3D MRI data with rigid body motion in all six degrees of freedom.

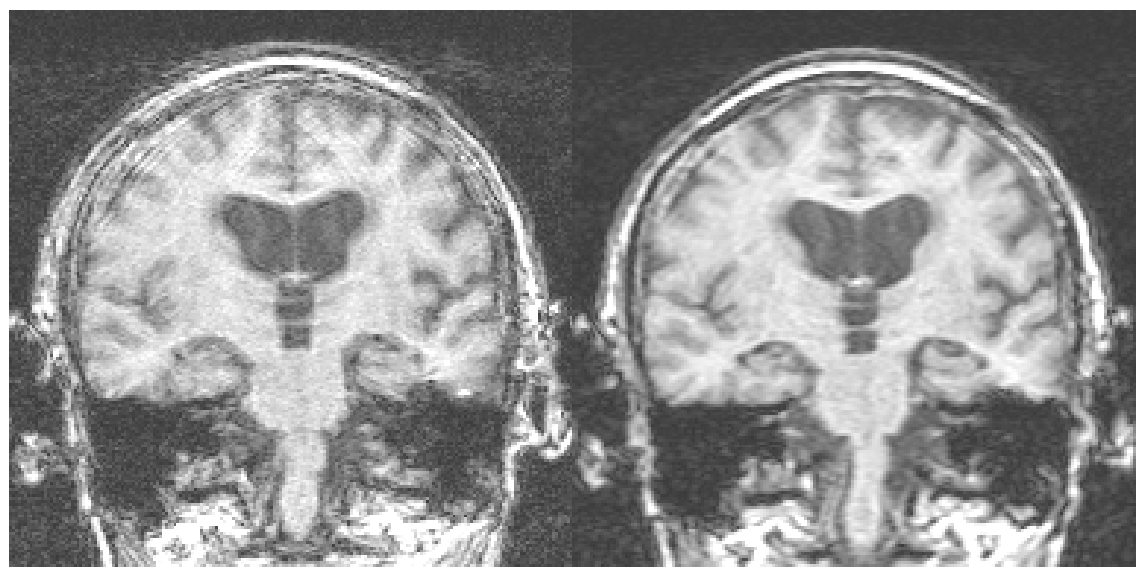

Fig. 1. A typical central slice of a data set with significant motion corruption before (left) and after (right) automatic motion correction.

\section{References}

Atkinson, D., Hill, D.L.G., Stoyle, P.N.R., Summers, P.E., Clare, S., Botwell, R., Keevil, S.F.: Automatic Compensation of Motion Artifacts in MRI. Mag. Res. Med. 41 (2000) 163-170

Manduca, A., McGee, K.P., Welch, E.B., Felmlee, J.P., Grimm, R.C. and Ehman, R.L.: Autocorrection in MR Imaging: Adaptive Motion Correction Without Navigator Echoes. Radiology 215 (2000) 904-909

Chen, B., Kaufman, A.: 3D Volume Rotation Using Shear Transformations. Graphical Models 62 (2000) 308-322 